\title{
Modified Variational Iteration Algorithm-II: Convergence and Applications to Diffusion Models
}

\author{
Hijaz Ahmad (D), ${ }^{1}$ Tufail A. Khan, ${ }^{1}$ Predrag S. Stanimirović, ${ }^{2}$ Yu-Ming Chu ${ }^{\text {D }},{ }^{3,4}$ \\ and Imtiaz Ahmad (iD ${ }^{5}$ \\ ${ }^{1}$ Department of Basic Sciences, University of Engineering and Technology, Peshawar, Pakistan \\ ${ }^{2}$ Faculty of Science and Mathematics, University of Niš, Višegradska 33, Niš 18000, Serbia \\ ${ }^{3}$ Department of Mathematics, Huzhou University, Huzhou 313000, China \\ ${ }^{4}$ Hunan Provincial Key Laboratory of Mathematical Modeling and Analysis in Engineering, University of Science \& Technology, \\ Changsha 410114, China \\ ${ }^{5}$ Department of Mathematics, University of Swabi, Swabi, Khyber Pakhtunkhwa, Pakistan
}

Correspondence should be addressed to Yu-Ming Chu; chuyuming2005@126.com

Received 10 September 2020; Accepted 21 September 2020; Published 6 October 2020

Academic Editor: Mostafa M. A. Khater

Copyright (C) 2020 Hijaz Ahmad et al. This is an open access article distributed under the Creative Commons Attribution License, which permits unrestricted use, distribution, and reproduction in any medium, provided the original work is properly cited.

\begin{abstract}
Variational iteration method has been extensively employed to deal with linear and nonlinear differential equations of integer and fractional order. The key property of the technique is its ability and flexibility to investigate linear and nonlinear models conveniently and accurately. The current study presents an improved algorithm to the variational iteration algorithm-II (VIA-II) for the numerical treatment of diffusion as well as convection-diffusion equations. This newly introduced modification is termed as the modified variational iteration algorithm-II (MVIA-II). The convergence of the MVIA-II is studied in the case of solving nonlinear equations. The main advantage of the MVIA-II improvement is an auxiliary parameter which makes sure a fast convergence of the standard VIA-II iteration algorithm. In order to verify the stability, accuracy, and computational speed of the method, the obtained solutions are compared numerically and graphically with the exact ones as well as with the results obtained by the previously proposed compact finite difference method and second kind Chebyshev wavelets. The comparison revealed that the modified version yields accurate results, converges rapidly, and offers better robustness in comparison with other methods used in the literature. Moreover, the basic idea depicted in this study is relied upon the possibility of the MVIA-II being utilized to handle nonlinear differential equations that arise in different fields of physical and biological sciences. A strong motivation for such applications is the fact that any discretization, transformation, or any assumptions are not required for this proposed algorithm in finding appropriate numerical solutions.
\end{abstract}

\section{Introduction}

Diffusion is a basic biofunction for all living organs; all nutrient materials are transferred to cells through biomembranes through a diffusion process [1]. It is quite important from the biological point of view because different processes such as exchange of gases, absorption of some substances in the gut, and absorption of water and minerals by the roots of the plants are examples of diffusion. The movement of substances between cells also involves diffusion. Cells are surrounded by a flexible and dynamic barrier known as a membrane. These biological membranes are composed of lipids, which aggregate to form a bilayer with particular biochemical properties. The amphipathic nature of the lipid bilayer, whose tails are hydrophobic and associated with each other and whose head groups are hydrophilic and interact with the aqueous environment, is critical to its structure. The composition of the lipid bilayer is also important for the diffusion both across and within the membrane. This membrane diffusion is important for a variety of functions, some of which include regulating the fluidity of the membrane, the uptake of metabolites into the 
cell from the outside, and the removal of waste products from the inside of the cell. The rate of diffusion is affected by properties of the cell, the surrounding solution, and the diffusing molecule. Simple equations and graphs can be used to examine how particular molecules and their concentration affect the rate of diffusion. Generally, the diffusion equation can be written as

$$
\frac{\partial \mathscr{Z}}{\partial t}=\frac{\partial}{\partial \xi}\left(D(\mathscr{Z}) \frac{\partial \mathscr{Z}}{\partial \xi}\right)+f(\xi, t),
$$

where $\mathscr{Z}$ is the concentration of the diffused material, for example, the concentration of the oxygen, $D$ is the diffusion coefficient, and $f$ is the source term. Equation (1) can also model spatial patterns of animals [2] and biological population [3]. Equation (1) can be rewritten in the following form:

$$
\frac{\partial \mathscr{Z}}{\partial t}=\frac{\partial D}{\partial \xi} \frac{\partial \mathscr{Z}}{\partial \xi}+D \frac{\partial^{2} \mathscr{Z}}{\partial \xi^{2}}+f(\xi, t) .
$$

Convection is the exchange of interior energy into or out of the body through the physical development of the encompassing liquid, which transports the inside energy and its mass. Since the piratical keep up their random motion, the total heat transfer is because of the superposition of the energy move coming about because of the random motion of the particles and the bulk motion of the fluid. It is customary to the utilization because of the transport due to the bulk amount of fluids. Convection can happen consequently freely if the fluid movement is caused by buoyancy, caused by a change in density, or caused by a change in the temperature of the fluid, or it can be imposed when the liquid is forced to flow over surface convection such as fans and pumps creating an artificially induced convection current. In numerous practical applications like heat loss from solar central receivers or cooling of PV modules, natural convection and forced convection occur at the same time.

More sophisticated models include diffusive and convective transport as key elements for modeling heat and mass transfer phenomena and continuous mechanical processes. It ought to be noticed that convective-diffusive transport is indispensable for predictions of various fluid and gas flows such as pollutants spreading in the atmosphere and pollutant transportation in groundwater and water basins [4]. Convection-diffusion problems are governed by typical mathematical models, which are common in gas and fluid dynamics. Mass and heat transfer takes place not only through diffusion but also through movement of the medium. Many scientists have considered different diffusion and convection-diffusion problems by different procedures; for example, the wavelet-Galerkin method was used by ElGamel [5], while the Bessel collocation method was implemented by Yüzbaşı and Sahin [6] for the solution of convection-diffusion problems. To investigate nonlinear diffusion problems, a nonclassical method has been presented by Saied [4], Wang [7] employed the Crank-Nicholson method, and Dhawan et al. [8] utilized the finite-element method.
For the convection-diffusion models, the piecewise-analytical method [9], high-order ADI method [10], leastsquares homotopy perturbation method [11], multigrid solver [12], lattice Boltzmann model [13], stabilized finiteelement method [14], second kind Chebyshev wavelets [15], finite element [16], finite difference method [17], decomposition method [18], compact finite difference method [19], meshless local Petrov-Galerkin method [20], discrete duality finite volume scheme [21], discontinuous Galerkin (DG) schemes [22], and high-order finite volume scheme [23] have been used in the literature.

Variational iteration method which was proposed originally by He [24] in 1999 has become popular in applied sciences and has been extensively employed by many researchers due to its promising performance in dealing with linear and nonlinear differential equations of integer and noninteger order. The key property of the technique is its ability and flexibility to investigate linear and nonlinear models conveniently and accurately. This technique has a simpler solution procedure and can be used to handle nonlinear differential equations that arise in different fields of science because any small discretization, Adomian polynomials, transformation, linearization, or any assumptions are not required for this method to find the numerical solutions [24-27]. So far, VIM has been utilized for the numerical and analytical investigations of fractional differential equations, oscillation equations, wave equations, some delay differential equations, etc., and can be utilized in a simplest way for the study of inverse problems [28], integrodifferential equations [29], differential-difference equations [30], and fractional calculus [31]. Many mathematicians have tried to develop this technique further, and so far many modifications have been introduced. Among these modifications, the modification where the leastsquares technology has been used by Herisanu and Marinca [32] is very interesting. Hesameddini and Latifizadeh [33] coupled it with Laplace transform, while its convergence was proved by Salkuyeh [34]. An auxiliary parameter was introduced by Inc and Yilmaz [35] to accelerate the convergence speed to the exact solution. In 2010, He et al. [25], the originator of the method himself, presented three algorithms, i.e., variational iteration algorithm-I (VIA-I), algorithm-II (VIA-II), and algorithm-III (VIA-III). The first author has modified the VIA-I successfully and implemented the modified version for many nonlinear PDEs $[36,37]$. Further, the first author also succeeded in modifying the algorithm-II by introducing an auxiliary parameter recently [38].

In this study, we aim to discuss the convergence analysis of the modified algorithm-II (MVIA-II) and to implement it for finding the numerical solution of nonlinear PDEs arising in physical and biological sciences which are modelled via the diffusion equations. We summarize the contents of the paper in the following sections. In Section 2, the proposed method and its implementation are described. A convergence analysis is discussed in Section 3. In Section 4, a nonlinear diffusion equation with two convection-diffusion models is investigated. Some concluding observations are discussed in the Section 5 . 


\section{Modified Iteration Algorithm-II}

In this section, the main idea of the modification is illustrated by considering a nonlinear differential equation:

$$
L[\mathscr{Z}(\xi)]+N[\mathscr{Z}(\xi)]=k(\xi),
$$

where $L[\mathscr{Z}(\xi)]$ and $N[\mathscr{Z}(\xi)]$ denote the linear and nonlinear operators, respectively, whereas $k(\xi)$ is a nonhomogeneous term. For an appropriate given initial condition $\mathscr{Z}_{0}(\xi)$, series solution $\mathscr{Z}_{n+1}(\xi)$ of equation (3) can be obtained as

$$
\mathscr{Z}_{n+1}(\xi)=\mathscr{Z}_{n}(\xi)+p \int_{0}^{\xi} \lambda(\vartheta)\left[L\left\{\mathscr{Z}_{n}(\vartheta)\right\}+\widetilde{N\left\{\mathscr{E}_{n}(\vartheta)\right\}}-k(\vartheta)\right] \mathrm{d} \vartheta,
$$

where $\overline{\mathscr{E}_{n}(\vartheta)}$ is a restricted term which gives $\delta \widetilde{\mathscr{\mathscr { E }}_{n}(\mathcal{\vartheta})}=0$ and $p$ and $\lambda(\vartheta)$ are the auxiliary parameter and Lagrange multiplier, respectively, which can be found optimally. The first one is used to accelerate the convergence to the exact solution [39-43], while the second one is used to construct the correction function [44]. The significant value of $\lambda(\vartheta)$ can be achieved by applying $\delta$ on both the sides of the recurrent relation (4) with respect to $\mathscr{Z}_{n}(\xi)$, which leads to

$$
\begin{aligned}
& \delta \mathscr{E}_{n+1}(\xi)=\delta \mathscr{X}_{n}(\xi) \\
& +p \delta \int_{0}^{\xi} \lambda(\vartheta)[L\left\{\mathscr{Z}_{n}(\vartheta)\right\}+\overbrace{N\left\{\mathscr{Z}_{n}(\vartheta)\right\}}-k(\vartheta)] \mathrm{d} \vartheta,
\end{aligned}
$$

where the following Lagrange multipliers can be obtained:

$$
\begin{aligned}
& \lambda=-1, \quad \text { for } i=1, \\
& \lambda=\xi-t, \quad \text { for } i=2 .
\end{aligned}
$$

Also, the following general formula for the Lagrange multiplier in the cases $i \geq 1$ is available:

$$
\lambda=\frac{(-1)^{i}(\vartheta-t)^{i-1}}{(i-1) !} .
$$

After finding the value of $\widetilde{\mathscr{E}_{n}(\vartheta)}$, an iteration formula is constructed by using this value in the correctional function 4 as follows:

$$
\begin{aligned}
\mathscr{Z}_{n+1}(\xi)= & \mathscr{Z}_{n}(\xi)+p \int_{0}^{\xi} \frac{(-1)^{i}(\vartheta-t)^{i-1}}{(i-1) !} \\
& {\left[L\left\{\mathscr{Z}_{n}(\vartheta)\right\}+N\left\{\mathscr{Z}_{n}(\vartheta)\right\}-k(\vartheta)\right] \mathrm{d} \vartheta . }
\end{aligned}
$$

A more summarizing and concise iteration formula that can be constructed is known as the modified variational iteration algorithm-II (MVIA-II):

$$
\begin{aligned}
\mathscr{Z}_{n+1}(\xi)= & \mathscr{Z}_{0}(\xi)+p \int_{0}^{\xi} \frac{(-1)^{i}(\vartheta-t)^{i-1}}{(i-1) !} \\
& \cdot\left[N\left\{\mathscr{Z}_{n}(\vartheta)\right\}-k(\vartheta)\right] \mathrm{d} \vartheta .
\end{aligned}
$$

The iterative sequence can be obtained starting from a proper initial approximation and using the iterative formula
(9). It is convenient to repeat iterations many times to arrive the given accuracy for the advanced computer technique. An exact solution $\mathscr{Z}(\xi)$ is obtained as

$$
\mathscr{Z}(\xi)=\lim _{n \longrightarrow \infty} \mathscr{Z}_{n}(\xi) \text {. }
$$

In this technique, one does not require the discretization of the domain and linearization of the given differential equations. We simply need to calculate the Lagrange multiplier of the given differential equation by restricting the nonlinear terms and get the analytical/numerical solution of the given differential equations in the series form:

$$
\left\{\begin{array}{l}
\mathscr{E}_{0}(\xi) \text { is an appropriate initial approximation, } \\
\mathscr{E}_{1}(\xi, p)=\mathscr{E}_{0}(\xi)+p \int_{0}^{\xi} \lambda(\vartheta)\left[L\left\{\mathscr{E}_{0}(\vartheta)\right\}+N\left\{\mathscr{E}_{0}(\vartheta)\right\}-k(\vartheta)\right] \mathrm{d} \vartheta \\
\mathscr{E}_{n+1}(\xi, p)=\mathscr{E}_{0}(\xi, p)+p \int_{0}^{\xi} \lambda(\vartheta)\left[N\left\{\mathscr{E}_{n}(\vartheta, p)\right\}-k(\vartheta, p)\right] \mathrm{d} \vartheta \\
n=1,2, \ldots
\end{array}\right.
$$

This procedure is known as the MVIA-II, where one does not require the discretization of the domain and linearization of the given differential equations. We employ this proposed procedure for finding the analytical/ numerical solution of diffusion and convection-diffusion equations. When $p=1$, the variational iteration algorithm given in equation (11) becomes the standard VIA-II, and the approximate solution converges to the exact one when $n$ approaches to infinity. Accordingly, a more accurate solution can be gained after a higher iteration process. Equation (11) has two obvious advantages: one is the limited step which is needed for better accuracy, while the other is an auxiliary parameter $(p)$ which can be optimally determined, and its value depends upon the iteration step $(n)$. When $n$ tends to infinity, the value of $p$ is equal to $p=1$. We give a criterion of how to suitably choose $n$ and optimally identify $p$ through examples. It can be seen clearly that the variation iteration algorithms are easy for the implementation. For nonlinear problems, the nonlinear terms have to be considered as restricted variations for obtaining the value of the Lagrange multiplier, and a correction functional can be easily constructed after determining the value identified value of corresponding nonlinear terms.

\section{Convergence Analysis}

This section is devoted to illustrate the convergence of the proposed method VIA-II having an auxiliary parameter for ensuring the convergence to an exact solution. This method can be implemented most readily in handling nonlinear differential equations, where one does not require any discretization of the domain or linearization of the given differential equations. The linear operator $L$ is defined as $L=$ $\partial^{2} / \partial t^{2}$ and $L=\partial / \partial t$, when it is employed to solve diffusion and convection-diffusion problems. During solving diffusion and convection-diffusion problems, the operator $R$ can be defined as 
$R \mathscr{Z}(\xi, t, p):=p \int_{0}^{t} \lambda(\vartheta)[L \mathscr{Z}(\xi, \vartheta, p)+\overbrace{N \mathscr{Z}(\xi, \vartheta, p)}-k(\vartheta)] \mathrm{d} \vartheta$

and $w_{n}$ and $v_{n}, n \geq 0$, are defined by

$$
\begin{aligned}
& \left\{\begin{array}{l}
w_{0}(\xi, t)=\mathscr{E}_{0}(\xi, t), \\
v_{0}(\xi, t)=w_{0}(\xi, t),
\end{array}\right. \\
& \left\{\begin{array}{l}
w_{1}(\xi, t, p)=R v_{0}(\xi, t), \\
v_{1}(\xi, t, p)=v_{0}(\xi, t)+R v_{0}(\xi, t),
\end{array}\right. \\
& \left\{\begin{array}{l}
\mathscr{X}_{1}(\xi, t, p)=R v_{0}(\xi, t), \\
v_{1}(\xi, t, p)=v_{0}(\xi, t)+w_{1}(\xi, t, p),
\end{array}\right. \\
& \left\{\begin{array}{l}
w_{n+1}(\xi, t, p)=R v_{n}(\xi, t, p), \\
v_{n+1}(\xi, t, p)=v_{n}(\xi, t, p)+R v_{n}(\xi, t, p) .
\end{array}\right.
\end{aligned}
$$

In general, for $n \geq 1$, it can be written as

$$
\left\{\begin{array}{l}
\mathscr{Z}_{n+1}(\xi, t, p)=R v_{n}(\xi, t, p), \\
v_{n+1}(\xi, t, p)=v_{n}(\xi, t, p)+w_{n+1}(\xi, t, p) .
\end{array}\right.
$$

Accordingly,

$$
\mathscr{Z}(\xi, t, p):=\lim _{n \longrightarrow \infty} v_{n}(\xi, t, p)=w_{0}(\xi, t)+\sum_{n=1}^{\infty} w_{n}(\xi, t, p) .
$$

$\mathscr{Z}_{0}(\xi, t)$ can be chosen uninhibitedly, but it needs to fulfil the corresponding initial-boundary conditions. The determination of appropriate initial approximation will give productive and accurate results. The $n$ th-order truncated series $\mathscr{Z}_{n}(\xi, t, p):=w_{0}(\xi, t)+\sum_{n=1}^{n} w_{n}(\xi, t, p)$ can be used to approximate the solution. The unknown parameter $p$ in $\mathscr{Z}_{n}(\xi, t, p)$ ensures that the hypothesis is fulfilled by utilizing 2 -norm error of the residual function. The error analysis and convergence criteria of VIA-II with an auxiliary parameter are revealed using the following theorems $[45,46]$.

Theorem 1. The operator $R$ defined in (12) maps a Hilbert space $H$ to $H$. The solution given in (15) in the form of series is as follows:

$$
\mathscr{Z}(\xi, t):=\lim _{n \longrightarrow \infty} v_{n}(\xi, t, p)=w_{0}(\xi, t)+\sum_{n=1}^{\infty} w_{n}(\xi, t, p) .
$$

It converges if $\exists p \neq 0,0<\beta<1$, such that

$$
\left\{\begin{array}{l}
\left\|R v_{0}(\xi, t)\right\| \leq \beta\left\|v_{0}(\xi, t)\right\|, \\
\left\|R v_{1}(\xi, t, p)\right\| \leq \beta\left\|R v_{0}(\xi, t)\right\|, \\
\left\|R v_{n}(\xi, t, p)\right\| \leq \beta\left\|R v_{n-1}(\xi, t, p)\right\|, \quad n=2,3,4, \ldots
\end{array}\right.
$$

Proof. To prove the required result, it is enough to verify that the sequence $\left\{v_{n}\right\}_{n=0}^{\infty}$ is a Cauchy sequence in the Hilbert space $H$. For that reason, we proceed as

$$
v_{0}(\xi, t)=w_{0}(\xi, t), v_{0+1}(\xi, t, p)=v_{0}(\xi, t)+R w_{0}(\xi, t)
$$

Further,

$$
v_{n+1}(\xi, t, p)=v_{n}(\xi, t, p)+R w_{n}(\xi, t, p),
$$

which implies

$$
\begin{aligned}
& \left\|v_{n+1}(\xi, t, p)-v_{n}(\xi, t, p)\right\|=\left\|R w_{n}(\xi, t, p)\right\| \\
& \leq \beta\left\|R w_{n-1}(\xi, t, p)\right\| \\
& \leq \beta^{2}\left\|R w_{n-2}(\xi, t, p)\right\| .
\end{aligned}
$$

Continuing in the same way, one obtains

$$
\left\|v_{n+1}(\xi, t, p)-v_{n}(\xi, t, p)\right\| \leq \beta^{n}\left\|R w_{0}(\xi, t, p)\right\| .
$$

Clearly, $\beta^{n} \longrightarrow 0$ as $n \longrightarrow \infty$. Thus,

$$
\left\|v_{n+1}(\xi, t, p)-v_{n}(\xi, t, p)\right\| \longrightarrow 0 .
$$

For every $n \geq i$, it holds that

$$
\left\|v_{n}-v_{i}\right\|=\left\|\left(v_{n}-v_{n-1}\right)+\left(v_{n-1}-v_{n-2}\right)+\cdots+\left(v_{i+1}-v_{i}\right)\right\|,
$$

which implies

$$
\left\|v_{n}-v_{i}\right\| \leq\left\|R w_{n-1}\right\|+\left\|R w_{n-2}\right\|+\cdots+\left\|R w_{j}\right\|,
$$

and later

$$
\begin{aligned}
\left\|v_{n}-v_{i}\right\| & \leq \beta^{n-1}\left\|R w_{0}\right\|+\beta^{n-2}\left\|R w_{n-2}\right\|+\cdots+\beta^{i}\left\|R w_{0}\right\| \\
& \leq\left(\beta^{n-1}+\beta^{n-2}+\cdots+\beta^{i}\right)\left\|R w_{0}\right\| \\
& =\frac{1-\beta^{n-i}}{1-\beta} \beta^{i+1}\left\|R w_{0}\right\| .
\end{aligned}
$$

As $0<\beta<1$, we get

$$
\lim _{n, i \longrightarrow \infty}\left\|v_{n}-v_{i}\right\|=0,
$$

which shows that the sequence $\left\{v_{n}\right\}_{n=0}^{\infty}$ is a Cauchy sequence in a Hilbert space $H$, which means that

$$
\mathscr{Z}(\xi, t)=\lim _{n \longrightarrow \infty} v_{n}(\xi, t, p)=w_{0}(\xi, t)+\sum_{n=1}^{\infty} w_{n}(\xi, t, p),
$$

converges.

Lemma 1. Let $Q$ be a function from a Hilbert space $H$ to $H$, the operator $L$ required in (3) be defined as $L=\partial^{i} / \partial t^{i}, i=1,2$, and the Lagrange multiplier be defined optimally by the variation theory; then,

$$
\left\{L \int_{0}^{t} \lambda(\vartheta) Q(\xi, \vartheta, p) \mathrm{d} \vartheta\right\}=-Q(\xi, \vartheta, p) .
$$


Proof. Consider the linear operator $L$ used in (3) defined as $L=\partial / \partial t$ and let $\lambda(\vartheta)=1$. Then,

$$
\begin{aligned}
\left\{L \int_{0}^{t} \lambda(\vartheta) Q(\xi, \vartheta, p) \mathrm{d} \vartheta\right\} & =\frac{\partial}{\partial t} \int_{0}^{t} \lambda(\vartheta) Q(\xi, \vartheta, p) \mathrm{d} \vartheta \\
& =-Q(\xi, \vartheta, p) .
\end{aligned}
$$

Similarly, let the operator $L$ required in (3) be defined as $L=\partial^{2} / \partial t^{2}$, and let $\lambda(\vartheta)=\vartheta-t$. Then,

$$
\begin{aligned}
& \left\{L \int_{0}^{t} \lambda(\vartheta) Q(\xi, \vartheta, p) \mathrm{d} \vartheta\right\}=\frac{\partial^{2}}{\partial t^{2}} \int_{0}^{t}(\vartheta-t) Q(\xi, \vartheta, p) \mathrm{d} \vartheta \\
& =-Q(\xi, \vartheta, p) \\
& =\frac{\partial}{\partial t}\left(\int_{0}^{t} \frac{\partial(\vartheta-t)}{\partial t} \mathrm{Q}(\xi, \vartheta, p) \mathrm{d} \vartheta+\left.(\vartheta-t) Q(\xi, \vartheta, p)\right|_{\vartheta=t}\right) \\
& =\frac{\partial}{\partial t} \int_{0}^{t}-Q(\xi, \vartheta, p) \mathrm{d} \vartheta=-Q(\xi, \vartheta, p),
\end{aligned}
$$

which verifies the statements.

Theorem 2. Let the operator $L$ needed in (3) be defined as $L=\partial^{i} / \partial t^{i}, i=1,2$. If we have the series solution (15) defined as

$$
\mathscr{Z}(\xi, t):=w_{0}(\xi, t)+\sum_{n=1}^{\infty} w_{n}(\xi, t, p),
$$

then $\mathscr{Z}(\xi, t)$ is an exact solution to the nonlinear partial differential equation (3).

Proof. Assume that the series solution (31) converges. Then, it implies the existence of $\lim _{n \rightarrow \infty} w_{n}(\xi, t, p)$ and

$$
\begin{aligned}
& {\left[w_{0}(\xi, t)-w_{1}(\xi, t, p)\right]+\sum_{j=1}^{n}\left[w_{j}(\xi, t, p)-w_{j+1}(\xi, t, p)\right]} \\
& =w_{0}(\xi, t)-w_{j+1}(\xi, t, p) .
\end{aligned}
$$

Therefore,

$$
\begin{aligned}
& {\left[w_{0}(\xi, t)-w_{1}(\xi, t, p)\right]+\sum_{j=1}^{\infty}\left[w_{j}(\xi, t, p)-w_{j+1}(\xi, t, p)\right]} \\
& =w_{0}(\xi, t)-\lim _{n \longrightarrow \infty} w_{n+1}(\xi, t, p) \\
& =w_{0}(\xi, t) .
\end{aligned}
$$

One can conclude from equation (15) that

$$
\mathscr{Z}(\xi, t)=w_{0}(\xi, t)+\sum_{n=1}^{\infty} w_{n}(\xi, t, p) .
$$

Applying the operator $L$ on both the sides, we get

$$
\begin{aligned}
& L\left[w_{0}(\xi, t)-w_{1}(\xi, t, p)\right]+\sum_{j=1}^{n} L\left[w_{j}(\xi, t, p)-w_{j+1}(\xi, t, p)\right] \\
& =L\left[w_{0}(\xi, t)\right]=0 .
\end{aligned}
$$

From Lemma 1 and definitions, it follows

$L\left[w_{0}(\xi, t)-w_{1}(\xi, t, p)\right]=L\left[w_{0}(\xi, t)\right]-L\left[R v_{0}(\xi, t)\right]$.

For simplicity, suppose

$$
M:=L \mathscr{Z}(\xi)+N \mathscr{Z}(\xi)-k(\xi) .
$$

It implies

$$
\begin{aligned}
L\left[w_{0}(\xi, t)-w_{1}(\xi, t, p)\right]= & -L\left[p \int_{0}^{t} \lambda(\vartheta) M v_{0}(\xi, t) \mathrm{d} \vartheta\right] \\
= & p M v_{0}(\xi, t), \\
L\left[w_{1}(\xi, t)-w_{2}(\xi, t, p)\right]= & L\left[w_{1}(\xi, t)\right]-L\left[R v_{2}(\xi, t)\right] \\
= & L\left[R v_{0}(\xi, t)\right]-L\left[R v_{1}(\xi, t, p)\right] \\
= & L\left[p \int_{0}^{t} \lambda(\vartheta) M v_{0}(\xi, t) \mathrm{d} \vartheta\right] \\
& -L\left[p \int_{0}^{t} \lambda(\vartheta) M v_{1}(\xi, t) \mathrm{d} \vartheta\right] \\
= & p\left[R v_{1}(\xi, t, p)-R v_{0}(\xi, t)\right] .
\end{aligned}
$$

In a similar way, for $j \geq 2$,

$L\left[w_{j}(\xi, t, p)-w_{j+1}(\xi, t, p)\right]=p\left[R v_{j}(\xi, t, p)-R v_{j-1}(\xi, t, p)\right]$.

Subsequently, it follows

$$
\begin{aligned}
L & {\left[w_{0}(\xi, t)-w_{1}(\xi, t, p)\right]+L\left[w_{1}(\xi, t, p)-w_{2}(\xi, t, p)\right] } \\
& +\sum_{j=2}^{n} L\left[w_{j}(\xi, t, p)-w_{j+1}(\xi, t, p)\right] \\
= & p M v_{0}(\xi, t)+p\left[R v_{1}(\xi, t, p)-R v_{0}(\xi, t)\right] \\
& +p\left[R v_{n}(\xi, t, p)-R v_{1}(\xi, t, p)\right] \\
= & p M v_{n}(\xi, t, p) \\
= & p M\left[w_{0}(\xi, t)+\sum_{j=1}^{n} w_{j}(\xi, t, p)\right] .
\end{aligned}
$$

Hence,

$$
\begin{aligned}
& L\left[w_{0}(\xi, t)-w_{1}(\xi, t, p)\right]+\sum_{j=1}^{n} L\left[w_{j}(\xi, t, p)-w_{j+1}(\xi, t, p)\right] \\
& \quad=p M\left[w_{0}(\xi, t)+\sum_{j=1}^{n} w_{j}(\xi, t, p)\right] .
\end{aligned}
$$

From equations (35) and (41), one can derive 


$$
p M\left[w_{0}(\xi, t)+\sum_{j=1}^{n} w_{j}(\xi, t, p)\right]=0 .
$$

Meanwhile, the unknown auxiliary parameter $p$ is a nonzero optimal number, and it is revealed that $\mathscr{Z}(\xi, t):=w_{0}(\xi, t)+\sum_{n=1}^{\infty} w_{n}(\xi, t, p)$ is an exact solution of the nonlinear partial differential equation (3), which was the required proof.

Theorem 3. Let us suppose that the solution $\mathscr{Z}(\xi, t):=w_{0}(\xi, t)+\sum_{n=1}^{\infty} w_{n}(\xi, t, p)$, given in (15), converges to the exact solution of the model equation (1). Also, assume that if the approximate solution is the truncated series $\mathscr{Z}_{N}(\xi, t):=w_{0}(\xi, t)+\sum_{n=1}^{N} w_{n}(\xi, t, p)$, then the maximum error norm can be assessed as

$$
\left\|\mathscr{Z}(\xi, t)-\mathscr{Z}_{N}(\xi, t)\right\| \leq\left\|\frac{1}{1-\beta} \beta^{N+1}\right\| \| w_{0}
$$

Proof. Following Theorem 1, one obtains

$$
\left\|v_{n}-v_{N}\right\| \leq \frac{1-\beta^{n-N}}{1-\beta} \beta^{N+1}\left\|w_{0}\right\| .
$$
follows

Since $n \longrightarrow 0$, then $v_{n}=\mathscr{Z}(\xi, t)$ and $0<\beta<1$, and it

$$
\left\|\mathscr{Z}(\xi, t)-\mathscr{Z}_{N}(\xi, t)\right\| \leq \frac{1}{1-\beta} \beta^{N+1}\left\|w_{0}\right\|,
$$

which completes the proof.

Shortly, defining

$$
\beta_{j}=\left\{\begin{array}{l}
\frac{\left\|w_{j+1}\right\|}{\left\|w_{j}\right\|}, \quad\left\|w_{j}\right\| \neq 0, \\
0, \quad\left\|w_{j}\right\|=0, \\
j=0,1,2, \ldots
\end{array}\right.
$$

At this point, if $0<\beta_{j}<1$ for $j=0,1,2, \ldots$, then $w_{0}(\xi, t)+$ $\sum_{n=1}^{\infty} w_{n}(\xi, t, p)$ of $(3)$ converges to the exact solution $\mathscr{Z}(\xi, t)$, whereas the maximum absolute error is equal to

$$
\left\|\mathscr{Z}(\xi, t)-\mathscr{Z}_{N}(\xi, t)\right\| \leq\left\|\frac{1}{1-\beta} \beta^{N+1}\right\| \| w_{0},
$$

where $\beta=\max \left\{\beta_{j}, j=0,1,2, \ldots\right\}$.

\section{Numerical Examples}

This section is dedicated to the numerical application of the proposed algorithm (MVIA-II) for different types of diffusion equations. Here, the approximate solutions to the diffusion as well as convection-diffusion equations are obtained effortlessly and smartly without any use of transformation or linearization. We assess the validity, efficiency, and accuracy of MVIA-II by solving different types of diffusion equations. The obtained approximate results are very significant and encouraging.

4.1. Test Problem 1. Consider the following nonlinear diffusion equation for the slow diffusion process [19]:

$$
\frac{\partial \mathscr{Z}}{\partial t}=\frac{\partial}{\partial \xi}\left(\mathscr{Z}^{2} \frac{\partial \mathscr{Z}}{\partial \xi}\right), \quad 0<\xi<1, t>0,
$$

with the initial condition:

$$
\mathscr{Z}(\xi, 0)=\frac{\xi+a}{2 c},
$$

where $a$ and $c$ are the arbitrary coefficients. The exact solution of equation (48) is

$$
\mathscr{Z}(\xi, t)=\frac{\xi+a}{2 \sqrt{c^{2}-t}}, \quad t<c^{2} .
$$

For our start, we solve the above system of the diffusion equation for the slow diffusion process by MVIA-II. The correction functional of MVIA-II for equation (48) is

$$
\begin{aligned}
& \mathscr{Z}_{n+1}(\xi, t, p)=\mathscr{Z}_{n}(\xi, t, p)+p \int_{0}^{t} \lambda(\xi) \\
& \{\frac{\partial \mathscr{E}_{n}(\xi, \vartheta, p)}{\partial t}-\overbrace{\frac{\partial}{\partial \xi}\left(\mathscr{E}_{n}(\xi, \vartheta, p)^{2} \frac{\partial \mathscr{E}_{n}(\xi, \vartheta, p)}{\partial \xi}\right)}\} \mathrm{d} \xi .
\end{aligned}
$$

The value of $\lambda(\xi)$ can be found with the help of the variational principle [47]. We find the value $\lambda(\xi)=-1$ of $\lambda(\xi)$. Usage of the obtained value of $\lambda(\xi)$ in (51) gives the below iterative formula:

$$
\begin{aligned}
\mathscr{Z}_{n+1}(\xi, t, p)= & \mathscr{Z}_{n}(\xi, t, p)-\int_{0}^{t}\left\{\frac{\partial \mathscr{Z}_{n}(\xi, \vartheta, p)}{\partial t}\right. \\
& \left.-\frac{\partial}{\partial \xi}\left(\mathscr{Z}_{n}(\xi, \vartheta, p)^{2} \frac{\partial \mathscr{Z}_{n}(\xi, \vartheta, p)}{\partial \xi}\right)\right\} \mathrm{d} \xi .
\end{aligned}
$$

A more summarizing and concise iteration formula can be constructed using the iterations (11):

$$
\begin{aligned}
\mathscr{E}_{n+1}(\xi, t, p)= & \mathscr{E}_{0}(\xi, t, p)-\int_{0}^{t}\left\{-\frac{\partial}{\partial \xi}\left(\mathscr{E}_{n}(\xi, \vartheta, p)^{2}\right.\right. \\
& \left.\left.\cdot \frac{\partial \mathscr{E}_{n}(\xi, \vartheta, p)}{\partial \xi}\right)\right\} \mathrm{d} \xi .
\end{aligned}
$$

Starting with a proper initial approximation,

$$
\mathscr{Z}_{0}(\xi, t)=\frac{\xi+a}{2 c} \text {. }
$$

Other iterations can be obtained by utilizing the iteration formula (53), by supposing $a=c=1$, as 


$$
\begin{aligned}
\mathscr{Z}_{1}(\xi, t, p) & =\frac{(p t+2)(\xi+1)}{4}, \\
\mathscr{Z}_{2}(\xi, t, p) & =\frac{\xi}{2}+\frac{p t(p t+4)(\xi+1)\left(p^{2} t^{2}+4 p t+8\right)}{128}+\frac{1}{2}, \\
& \ldots .
\end{aligned}
$$

The threshold value of $n$ for this case is $n=4$. To find a proper value of $p$ for $\mathscr{Z}_{4}(\xi, t, p)$, the following residual function is defined:

$$
r_{4}(\xi, t, p)=\frac{\partial \mathscr{E}_{4}(\xi, t, p)}{\partial t}-\frac{\partial}{\partial \xi}\left(\frac{1}{\mathscr{E}_{4}(\xi, t, p)^{2}} \frac{\partial \mathscr{E}_{4}(\xi, t, p)}{\partial \xi}\right) .
$$

The 2-norm of the residual function (56) in the 4th iteration with respect to $p$ for $(\xi, t) \in[0,1] \times[0,1]$ is

$$
e_{4}(p)=\left[\frac{1}{(11)^{2}} \sum_{i=0}^{10} \sum_{j=0}^{10}\left[r_{4}\left(\frac{i}{10}, \frac{j}{100}, p\right)\right]^{2}\right]^{1 / 2} \text {. }
$$

The residual function (56) can be used to approximate $e_{4}(p)$, while the optimal value of $p$ can be determined by minimizing $e_{4}(p)$. The value of $p$ is equal to 1.00000601028068 when the minimum value $3.21205524320124664^{*} 10^{-6}$ of $e_{4}(p)$ is reached. In this example, we choose a good initial approximation, and the value of $p$ is near 1 , showing the initial approximation is of great importance for a fast convergence. Results are obtained with the usage of this optimal value of $p$ in $\mathscr{X}_{4}(\xi, t, p)$ in the space-time domain $(\xi, t) \in[0,1] \times[0,1]$. Further, to show how the numerical solution converges to the exact solution, we compute $\beta_{n}$ values for the considered nonlinear diffusion problem:

$$
\begin{aligned}
& \beta_{0}=\frac{\left|\mathscr{E}_{1}(\xi, t, p)\right|}{\left|\mathscr{E}_{0}(\xi, t, p)\right|}=0.150000901542102<1, \\
& \beta_{1}=\frac{\left|\mathscr{E}_{2}(\xi, t, p)\right|}{\left|\mathscr{E}_{1}(\xi, t, p)\right|}=0.032393045990139<1, \\
& \beta_{2}=\frac{\left|\mathscr{E}_{3}(\xi, t, p)\right|}{\left|\mathscr{E}_{2}(\xi, t, p)\right|}=0.005810185025718<1,
\end{aligned}
$$

Obtained values show that for $n \geq 0$, the values $\beta_{n}$ are less than one, which provide the proof as well that the proposed MVIA-II algorithm is convergent.

Showing the effectiveness of the algorithm, numerical results of the proposed method for Test Problem 4.1 are reported in Table 1 for various values of $x$ and $t$. It can be observed from the table that the results of the proposed algorithm match with the exact solution and with the results reported for CFD6 [19] as well. We have also compared the
TABle 1: The comparison of results for Test Problem 4.1 for different values of $t$ and $\xi$.

\begin{tabular}{lcccccc}
\hline & & \multicolumn{2}{c}{ Approximate } & \multicolumn{2}{c}{ CPU time in } \\
$t$ & $\xi$ & $\begin{array}{c}\text { Exact } \\
\text { seconds }\end{array}$ & \multicolumn{2}{c}{ solution } & \multicolumn{2}{c}{ selution } \\
& & & Present & CFD6 [19] & Present & CFD6 [19] \\
\hline \multirow{4}{*}{01} & 0.1 & 0.552771 & 0.552771 & 0.552771 & & \\
& 0.5 & 0.753778 & 0.753778 & 0.753778 & 0.050035 & 0.09 \\
& 0.9 & 0.954786 & 0.954786 & 0.954786 & & \\
\hline & 0.1 & 0.579751 & 0.579751 & 0.579751 & & \\
0.1 & 0.5 & 0.790569 & 0.790569 & 0.790569 & 0.088120 & 0.38 \\
& 0.9 & 1.001388 & 1.001388 & 1.001388 & & \\
\hline
\end{tabular}

CPU time and found that MVIA-II is faster than the compact finite difference method (CFD6) [19].

The approximate solution for different times $t=0.01$, $t=0.05, t=0.10, t=0.20$, and $t=0.30$ is visualized in Figure 1 for Test Problem 4.1.

Comparative analysis of the proposed MVIA-II with respect to CDF6 in terms of spatial convergence rate is given in Table 2. It can be seen from this table that the MVIA-II is more accurate than the CDF6 [19].

4.2. Test Problem 2. Consider the convection-diffusion equation from [15] of the following form:

$$
\frac{\partial \mathscr{Z}}{\partial t}+a(\xi) \frac{\partial \mathscr{E}}{\partial \xi}=b(\xi) \frac{\partial^{2} \mathscr{Z}}{\partial \xi^{2}}+f(\xi, t),
$$

with initial/boundary conditions:

$$
\mathscr{Z}(\xi, 0)=\xi^{3}, \mathscr{Z}(0, t)=0, \mathscr{Z}(1, t)=e^{t} .
$$

The exact solution of equation (59) is

$$
\mathscr{Z}(\xi, t)=\xi^{3} e^{t} .
$$

For our start, we solve the system of the convectiondiffusion equation by the MVIA-II, and for comparison purposes, we take $a(\xi)=-\xi / 6, b(\xi)=\xi^{2} / 12$, and $f(\xi, t)=0$. Constructing the correction functional of MVIA-II for equation (59) as

$$
\begin{aligned}
& \mathscr{X}_{n+1}(\xi, t, p)=\mathscr{X}_{n}(\xi, t, p)+p \int_{0}^{t} \lambda(\xi) \\
& \left\{\frac{\partial \mathscr{E}_{n}(\xi, \vartheta, p)}{\partial t}-\frac{x}{6} \overline{\frac{\partial \mathscr{X}_{n}(\xi, \vartheta, p)}{\partial \xi}}-\frac{x^{2}}{12} \overline{\frac{\partial^{2} \mathscr{I}_{n}(\xi, \vartheta, p)}{\partial \xi^{2}}}\right\} \mathrm{d} \xi .
\end{aligned}
$$

The value of $\lambda(\xi)$ may be determined easily with the help of the variational theory:

$$
\begin{aligned}
& \delta \mathscr{E}_{n+1}(\xi, t, p)=\delta \mathscr{E}_{n}(\xi, t, p)+\delta p \int_{0}^{t} \\
& \lambda(\xi)\left\{\frac{\partial \mathscr{E}_{n}(\xi, \vartheta, p)}{\partial t}-\frac{x}{6} \frac{\overline{\partial \mathscr{E}_{n}(\xi, \vartheta, p)}}{\partial \xi}-\frac{x^{2}}{12} \overline{\frac{\partial^{2} \mathscr{E}_{n}(\xi, \vartheta, p)}{\partial \xi^{2}}}\right\} \mathrm{d} \xi .
\end{aligned}
$$

Ignoring the restricted terms, 


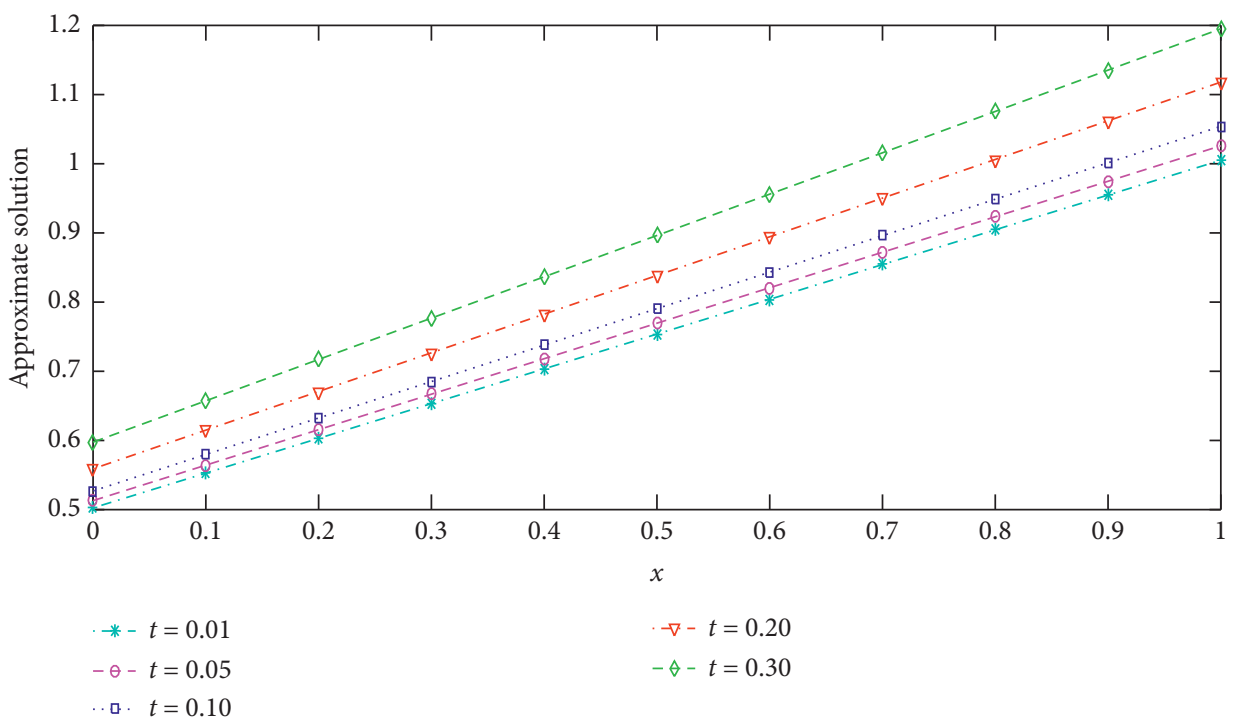

Figure 1: The results of MVIA-II for Test Problem 4.1 for different values of time.

TABLE 2: Comparison of convergence rates for $t=0.001$ for Test Problem 4.1.

\begin{tabular}{lcc}
\hline$h$ & CFD6 [19] & Present method \\
\hline $1 / 8$ & $3 .-e-07$ & $3.0-e-09$ \\
$1 / 10$ & $3 .-e-07$ & $3.0-e-09$ \\
$1 / 12$ & $3 .-e-07$ & $3.0-e-09$ \\
$1 / 14$ & $3 .-e-07$ & $3.0-e-09$ \\
\hline
\end{tabular}

$$
\begin{aligned}
\delta \mathscr{E}_{n+1}(\xi, t, p) & =\delta \mathscr{E}_{n}(\xi, t, p)+p \delta \int_{0}^{t} \lambda(\xi)\left\{\frac{\partial \mathscr{E}_{n}(x, \xi, p)}{\partial \xi}\right\} \mathrm{d} \xi \\
& =(1+\lambda(\xi)) \delta \mathscr{E}_{n}(\xi, t, p)-p \int_{0}^{t} \lambda^{\prime}(\xi) \delta \mathscr{E}_{n}(\xi, \vartheta, p) \mathrm{d} \xi,
\end{aligned}
$$

corresponding stationary conditions are $\lambda^{\prime}(\xi)=0$ and $1+\lambda(\xi)=0$, and we obtain the value of $\lambda(\xi)$ which is $\lambda(\xi)=-1$. Using this obtained value of $\lambda(\xi)$ in equation (62) gives the below iterative iteration formula:

$$
\begin{aligned}
\mathscr{Z}_{n+1}(\xi, t, p)= & \mathscr{E}_{n}(\xi, t, p) \\
& -\int_{0}^{t}\left\{\frac{\partial \mathscr{E}_{n}(\xi, \vartheta, p)}{\partial t}-\frac{x}{6} \frac{\partial \mathscr{E}_{n}(\xi, \vartheta, p)}{\partial \xi}\right. \\
& \left.-\frac{x^{2}}{12} \frac{\partial^{2} \mathscr{E}_{n}(\xi, \vartheta, p)}{\partial \xi^{2}}\right\} \mathrm{d} \xi .
\end{aligned}
$$

A more concise iteration can be constructed using the iterative formula (11):

$$
\begin{aligned}
& \mathscr{Z}_{n+1}(\xi, t, p)=\mathscr{E}_{0}(\xi, t, p) \\
& \quad-\int_{0}^{t}\left\{-\frac{x}{6} \frac{\partial \mathscr{X}_{n}(\xi, \vartheta, p)}{\partial \xi}-\frac{x^{2}}{12} \frac{\partial^{2} \mathscr{X}_{n}(\xi, \vartheta, p)}{\partial \xi^{2}}\right\} \mathrm{d} \xi .
\end{aligned}
$$

Starting with a proper initial approximation,

$$
\mathscr{Z}_{0}(\xi, t)=\xi^{3} .
$$

Other iterations can be obtained by utilizing the iteration formula (66):

$$
\begin{aligned}
& \mathscr{Z}_{1}(\xi, t, p)=\xi^{3}(p t+1), \\
& \mathscr{I}_{2}(\xi, t, p)=\xi^{3}+\frac{\left(p t \xi^{3}(p t+2)\right)}{6}, \\
& \mathscr{Z}_{3}(\xi, t, p)=\xi^{3}+\frac{p t \xi^{3}\left(p^{2} t^{2}+3 p t+6\right)}{6},
\end{aligned}
$$

The threshold value of $n$ is $n=10$ and is used to find a proper value of $p$ for $\mathscr{Z}_{5}(\xi, t, p)$. The following residual function is defined:

$$
r_{10}(\xi, t, p)=\frac{\partial \mathscr{E}_{1} 0(\xi, t, p)}{\partial t}-\frac{\partial}{\partial \xi}\left(\frac{1}{\mathscr{E}_{10}(\xi, t, p)^{2}} \frac{\partial \mathscr{E}_{1} 0(\xi, t, p)}{\partial \xi}\right) .
$$

The 2-norm of residual function (69) for the 4th iteration with respect to $p$ for $(\xi, t) \in[0,1] \times[0,1]$ is

$$
e_{10}(p)=\left[\frac{1}{(11)^{2}} \sum_{i=0}^{10} \sum_{j=0}^{10}\left[r_{10}\left(\frac{i}{10}, \frac{j}{10}, p\right)\right]^{2}\right]^{1 / 2} .
$$

The residual function (69) is utilized to approximate $e_{5}(p)$ for finding the optimal value of $p$, which can be found by minimizing $e_{4}(p)$. The value of $p$ is found to be 1.00000002955466 when the minimum value of $e_{4}(p)$ is $2.98660438462132656^{*} 10^{-8}$. With the usage of this optimal 


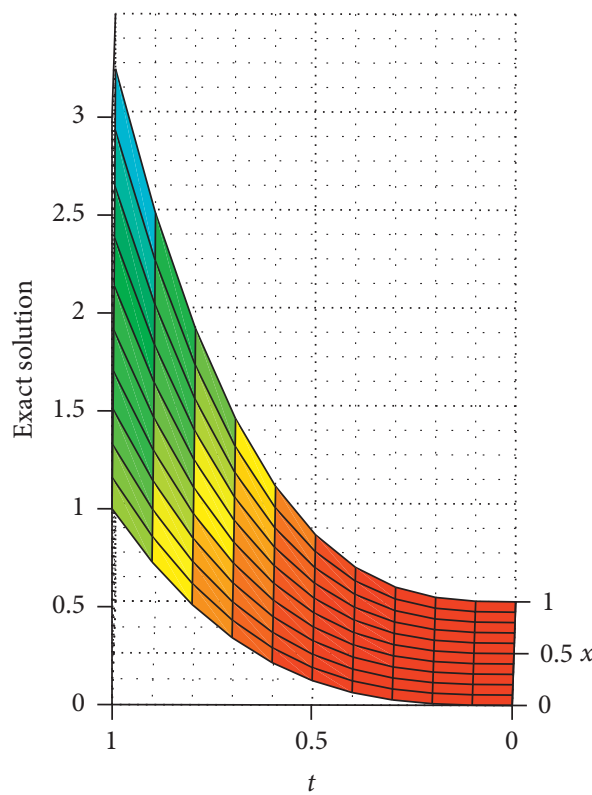

(a)

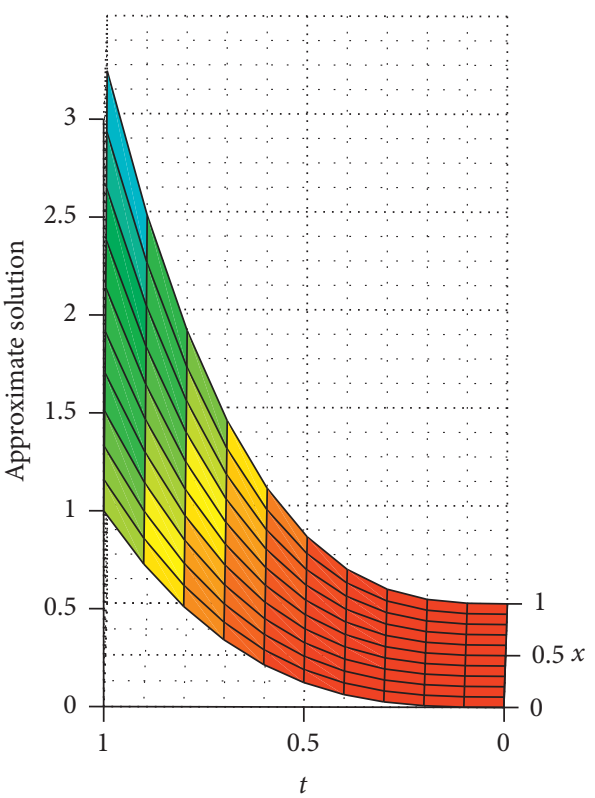

(b)

FIgURE 2: Solution behavior of (a) exact and (b) approximate solutions for Test Problem 4.2.

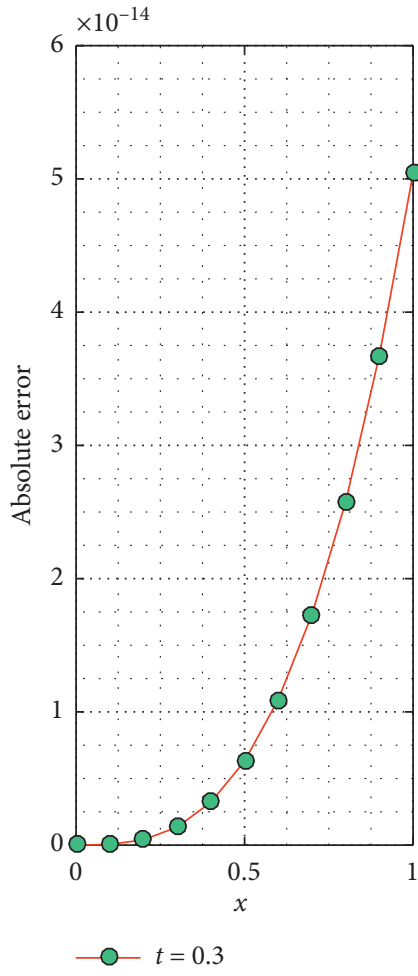

(a)

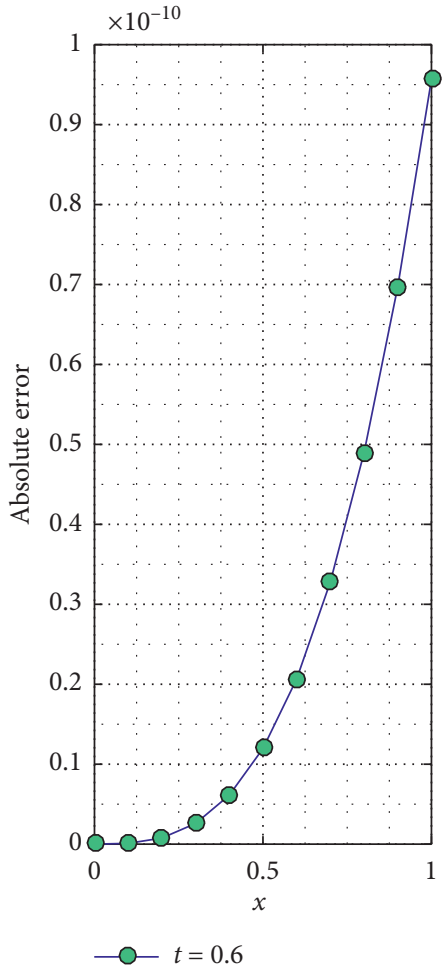

(b)

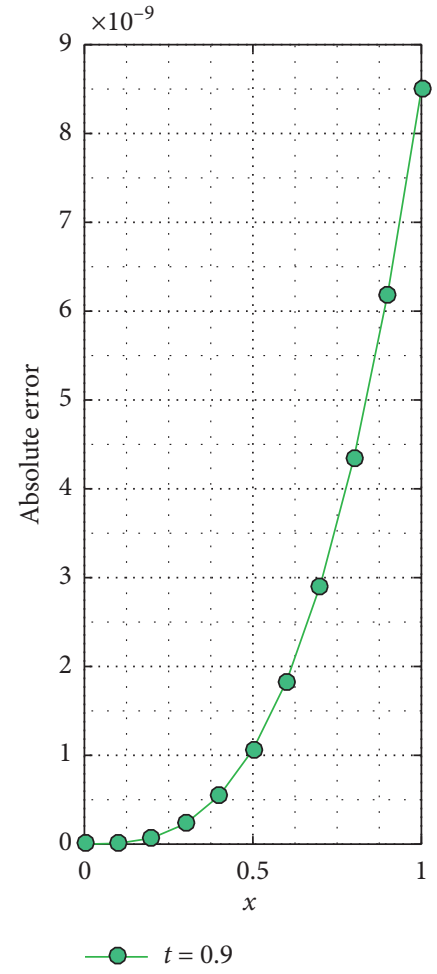

(c)

Figure 3: Comparison in terms of absolute errors for Test Problem 4.2.

value of $p$ in $\mathscr{X}_{10}(\xi, t, p)$ in the space-time domain $(\xi, t) \in[0,1] \times[0,1]$, the behavior of exact and present solutions can be perceived in Figures 2 and 3.
Further, to show how the numerical solution converges to an exact solution, we compute $\beta_{n}$ values for this nonlinear diffusion problem: 


$$
\begin{aligned}
& \beta_{0}=\frac{\left|\mathscr{Z}_{1}(\xi, t, p)\right|}{\left|\mathscr{E}_{0}(\xi, t, p)\right|}=0.300001803084204<1, \\
& \beta_{1}=\frac{\left|\mathscr{E}_{2}(\xi, t, p)\right|}{\left|\mathscr{E}_{1}(\xi, t, p)\right|}=0.034615752701361<1, \\
& \beta_{2}=\frac{\left|\mathscr{Z}_{3}(\xi, t, p)\right|}{\left|\mathscr{Z}_{2}(\xi, t, p)\right|}=0.003345779402775<1, \\
& \beta_{3}=\frac{\left|\mathscr{Z}_{4}(\xi, t, p)\right|}{\left|\mathscr{Z}_{3}(\xi, t, p)\right|}=0.000250098190015<1,
\end{aligned}
$$

which show that for $n \geq 0$, the values of $\beta_{n}$ are less than one, which provide the proof as well that the MVIA-II is convergent.

In Table 3, we compare the results generated by the MVIA-II with the exact one and with the method reported in [48] for Test Problem 4.2. From this table, we can conclude that the proposed algorithm produces better results than the results of a technique based on Hermite interpolant multiscaling functions given in [48].

The behavior of the exact and approximate solutions obtained by the MVIA-II is given in Figure 2. It can be revealed from this figure that the MVIA-II provides accurate and precise results. The absolute error for times $t=0.3$, $t=0.6$, and $t=0.9$ is shown in Figure 3 , and superb accuracy has been archived in this case as well.

4.3. Test Problem 3. Consider the convection-diffusion equation from [48] of the following form:

$$
\frac{\partial \mathscr{Z}}{\partial t}+a(\xi) \frac{\partial \mathscr{Z}}{\partial \xi}=b(\xi) \frac{\partial^{2} \mathscr{Z}}{\partial \xi^{2}}+f(\xi, t),
$$

with initial/boundary conditions:

$$
\mathscr{Z}(\xi, 0)=e^{-\xi}, \mathscr{Z}(0, t)=e^{-0.09 t}, \mathscr{Z}(1, t)=e^{-1-0.09 t} .
$$

The exact solution of equation (72) is

$$
\mathscr{Z}(\xi, t)=e^{-\xi-0.09 t} \text {. }
$$

For our start, we solve the convection-diffusion equation by the MVIA-I, and for comparison purposes, we use $a(\xi)=-0.1, b(\xi)=0.01$, and $f(\xi, t)=0$. Constructing the correction functional of MVIA-II for equation (72) as

$$
\begin{aligned}
& \mathscr{X}_{n+1}(\xi, t, p)=\mathscr{E}_{n}(\xi, t, p)+p \int_{0}^{t} \lambda(\xi) \\
& \quad\left\{\frac{\partial \mathscr{E}_{n}(\xi, \vartheta, p)}{\partial t}-0.1 \overline{\frac{\partial \mathscr{E}_{n}(\xi, \vartheta, p)}{\partial \xi}}-0.01 \overline{\frac{\partial^{2} \mathscr{E}_{n}(\xi, \vartheta, p)}{\partial \xi^{2}}}\right\} \mathrm{d} \xi .
\end{aligned}
$$

The value of $\lambda(\xi)$ may be determined easily with the help of the variational theory:

$$
\begin{aligned}
& \delta \mathscr{E}_{n+1}(\xi, t, p)=\delta \mathscr{E}_{n}(\xi, t, p)+\delta p \int_{0}^{t} \lambda(\xi) \\
& \quad\left\{\frac{\partial \mathscr{E}_{n}(\xi, \vartheta, p)}{\partial t}-0.1 \frac{\widetilde{\partial \mathscr{E}_{n}(\xi, \vartheta, p)}}{\partial \xi}-0.01 \overline{\frac{\partial^{2} \mathscr{E}_{n}(\xi, \vartheta, p)}{\partial \xi^{2}}}\right\} \mathrm{d} \xi .
\end{aligned}
$$

Ignoring the restricted terms, the stationary conditions are $\lambda^{\prime}(\xi)=0$ and $1+\lambda(\xi)=0$, from which we obtain the value $\lambda(\xi)=-1$ of $\lambda(\xi)$. Using this obtained value of $\lambda(\xi)$, equation (75) gives the below iterative formula:

$$
\begin{aligned}
& \mathscr{Z}_{n+1}(\xi, t, p)=\mathscr{Z}_{n}(\xi, t, p) \\
& \quad-\int_{0}^{t}\left\{\frac{\partial \mathscr{E}_{n}(\xi, \vartheta, p)}{\partial t}-0.1 \frac{\partial \mathscr{E}_{n}(\xi, \vartheta, p)}{\partial \xi}-0.01 \frac{\partial^{2} \mathscr{Z}_{n}(\xi, \vartheta, p)}{\partial \xi^{2}}\right\} \mathrm{d} \xi .
\end{aligned}
$$

A more summarizing iteration can be constructed using the iterative formula (11):

$$
\begin{aligned}
& \mathscr{Z}_{n+1}(\xi, t, p)=\mathscr{Z}_{0}(\xi, t, p)-p \int_{0}^{t} \\
& \quad \cdot\left\{-0.1 \frac{\partial \mathscr{E}_{n}(\xi, \vartheta, p)}{\partial \xi}-0.01 \frac{\partial^{2} \mathscr{E}_{n}(\xi, \vartheta, p)}{\partial \xi^{2}}\right\} \mathrm{d} \xi .
\end{aligned}
$$

Starting with a proper initial approximation,

$$
\mathscr{Z}_{0}(\xi, t)=e^{-\xi} \text {. }
$$

The other iterations can be obtained by using the iterative scheme (78), as

$$
\begin{aligned}
& \mathscr{Z}_{1}(\xi, t, p)=-\frac{e^{-\xi}(9 p t-100)}{100}, \\
& \mathscr{Z}_{2}(\xi, t, p)=-\frac{e^{-\xi}\left(81 p^{2} t^{2}+1800 p t+20000\right)}{20000},
\end{aligned}
$$

The threshold value of $n$ in this example is $n=5$ and is used to find a proper value of $p$ for $\mathscr{Z}_{5}(\xi, t, p)$. The following residual function is defined:

$$
r_{6}(\xi, t, p)=\frac{\partial \mathscr{E}_{6}(\xi, t, p)}{\partial t}-0.1 \frac{\partial \mathscr{Z}_{6}(\xi, t, p)}{\partial \xi}-0.01 \frac{\partial^{2} \mathscr{E}_{6}(\xi, t, p)}{\partial \xi^{2}} .
$$

The 2-norm of residual function (81) for the 6th iteration with respect to $p$ for $(\xi, t) \in[0,1] \times[0,1]$ is equal to

$$
e_{6}(p)=\left[\frac{1}{(11)^{2}} \sum_{i=0}^{10} \sum_{j=0}^{10}\left[r_{6}\left(\frac{i}{10}, \frac{j}{10}, p\right)\right]^{2}\right]^{1 / 2} \text {. }
$$


TABle 3: For different values of $t$ and $\xi$, comparison of results for Test Problem 4.2.

\begin{tabular}{|c|c|c|c|c|c|}
\hline \multirow{2}{*}{$\xi$} & \multirow{2}{*}{ Exact solution } & \multirow{2}{*}{ Present method } & \multicolumn{3}{|c|}{ Method of [48] } \\
\hline & & & $J=0$ & $J=1$ & $J=2$ \\
\hline 0.1 & 0.001349859 & 0.001349859 & 0.001349859 & 0.001349859 & 0.001349859 \\
\hline 0.2 & 0.010798870 & 0.010798870 & 0.010798870 & 0.010798870 & 0.01079887 \\
\hline 0.3 & 0.036446188 & 0.036446188 & 0.036446185 & 0.036446188 & 0.036446188 \\
\hline 0.4 & 0.086390964 & 0.086390964 & 0.086390958 & 0.086390964 & 0.086390964 \\
\hline 0.5 & 0.168732351 & 0.168732351 & 0.168732340 & 0.168732351 & 0.168732351 \\
\hline 0.6 & 0.291569502 & 0.291569502 & 0.291569483 & 0.291569502 & 0.291569502 \\
\hline 0.7 & 0.463001571 & 0.463001571 & 0.463001541 & 0.463001571 & 0.463001571 \\
\hline 0.8 & 0.691127709 & 0.691127709 & 0.691127665 & 0.691127709 & 0.691127709 \\
\hline 0.9 & 0.984047071 & 0.984047071 & 0.984047010 & 0.984047070 & 0.984047071 \\
\hline 1.0 & 1.349858808 & 1.349858808 & 1.349858729 & 1.349858807 & 1.349858808 \\
\hline
\end{tabular}

Table 4: Comparison of solutions for various values of $\xi$ for Test Problem 4.3.

\begin{tabular}{|c|c|c|c|c|c|}
\hline \multirow{2}{*}{$\xi$} & \multirow{2}{*}{ Exact solution } & \multirow{2}{*}{ Approximate solution } & \multicolumn{3}{|c|}{ Absolute errors } \\
\hline & & & Present method & {$[48]$} & {$[15]$} \\
\hline 0.1 & 0.880733672597157 & 0.880733672597159 & $1.7764 e-15$ & $1.03 e-13$ & $4.65 e-12$ \\
\hline 0.2 & 0.796920782290140 & 0.796920782290141 & $1.6653 e-15$ & $2.11 e-13$ & $8.34 e-12$ \\
\hline 0.3 & 0.721083743026607 & 0.721083743026608 & $1.5543 e-15$ & $4.13 e-13$ & $1.36 e-11$ \\
\hline 0.4 & 0.652463552227900 & 0.652463552227902 & $1.4433 e-15$ & $4.36 e-13$ & $3.85 e-11$ \\
\hline 0.5 & 0.590373435960464 & 0.590373435960465 & $1.3323 e-15$ & $3.70 e-13$ & $7.53 e-10$ \\
\hline 0.6 & 0.534191975471484 & 0.534191975471485 & $1.2212 e-15$ & $3.85 e-13$ & $9.90 e-12$ \\
\hline 0.7 & 0.483356887821146 & 0.483356887821147 & $9.9920 e-16$ & $7.44 e-14$ & $5.54 e-12$ \\
\hline 0.8 & 0.437359398365983 & 0.437359398365984 & $9.4369 e-16$ & $1.00 e-13$ & $1.18 e-11$ \\
\hline 0.9 & 0.395739148771237 & 0.395739148771238 & $8.3267 e-16$ & $3.20 e-13$ & $7.69 e-11$ \\
\hline
\end{tabular}

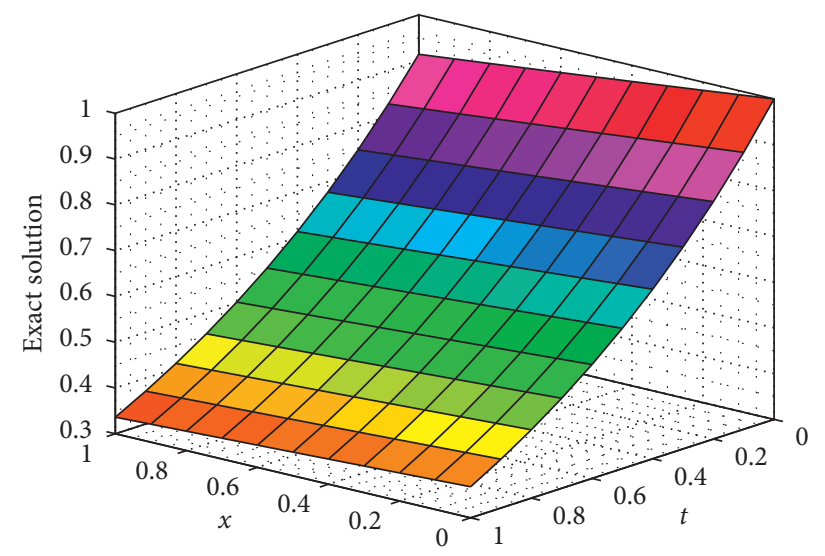

(a)

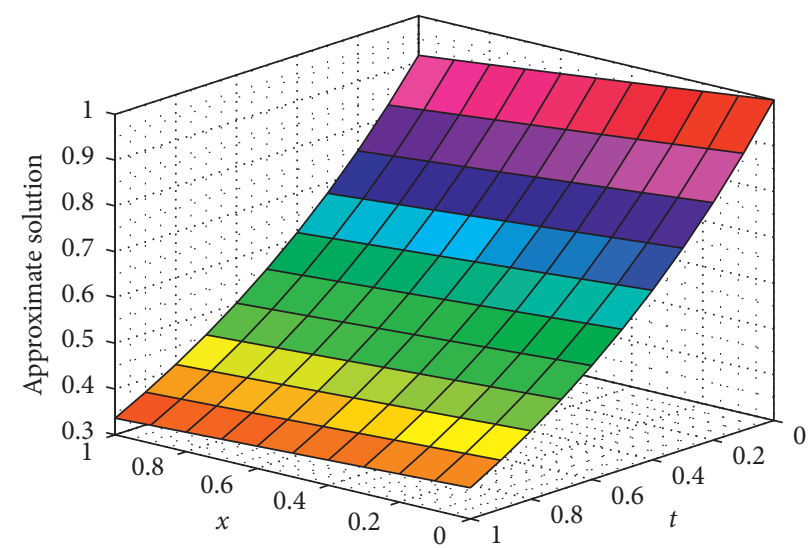

(b)

FIgURE 4: Solution behavior graphs of (a) exact and (b) approximate solutions for Test Problem 4.3.

The residual function (81) is used to approximate $e_{6}(p)$ for finding the optimal value of $p$, which can be found by minimizing $e_{6}(p)$. The value of $p$ is found to be 1 when the minimum value of $e_{6}(p)$ is $2.38042263784292823^{*} 10^{-22}$. In this example, we choose a good initial approximation, and the value of $p$ is 1 , showing that the initial approximation is of great importance for fast convergence. With the usage of this optimal value of $p$ in $\mathscr{Z}_{4}(\xi, t, p)$ in the space-time domain $(\xi, t) \varepsilon[0,1] \times[0,1]$, the approximate solution is obtained. Further, 


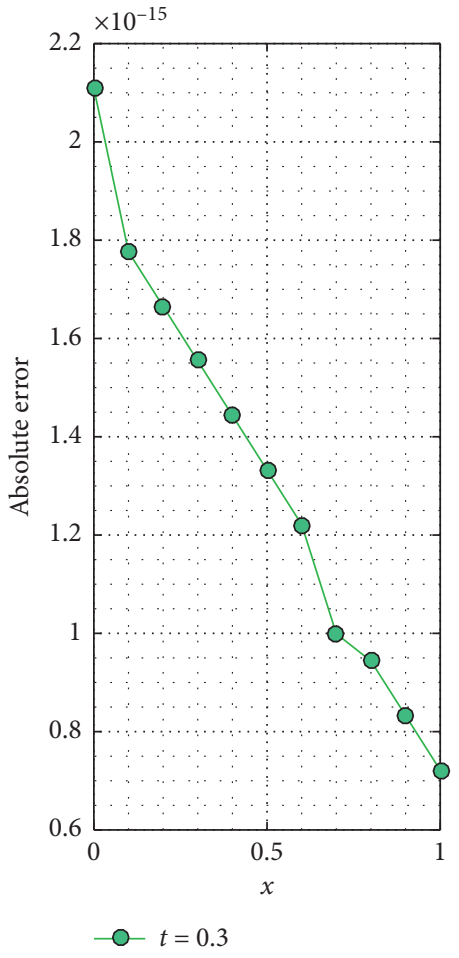

(a)

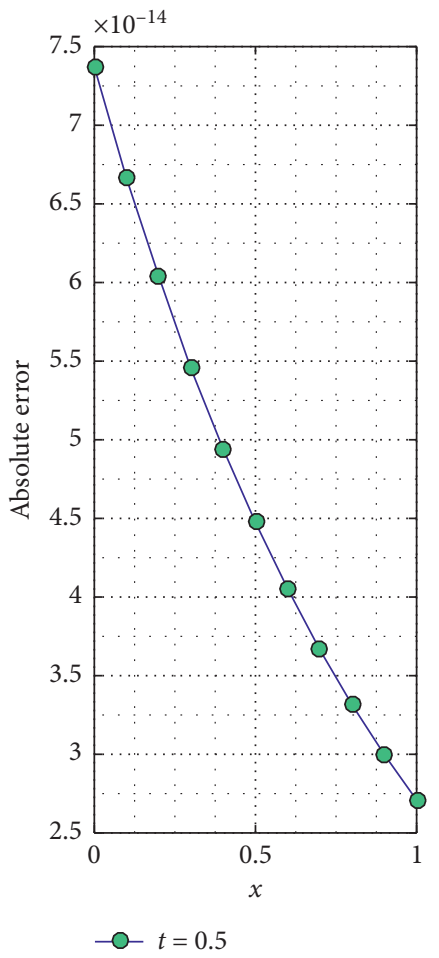

(b)

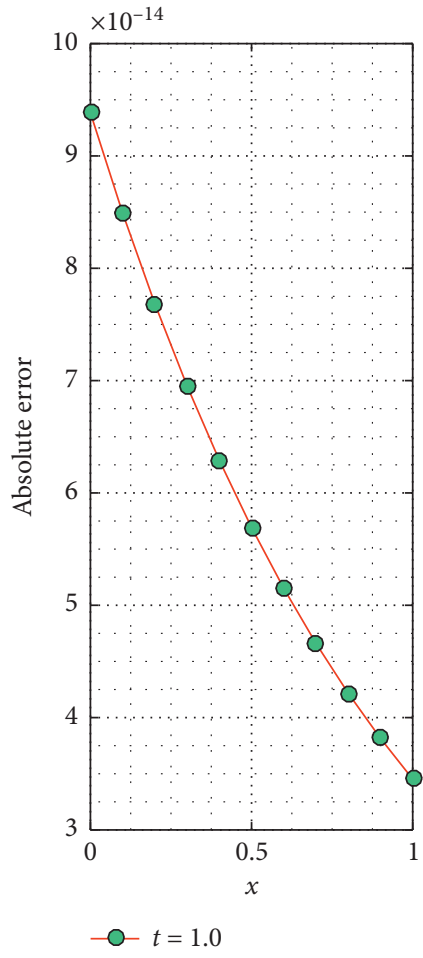

(c)

Figure 5: Comparison in terms of absolute errors for various values of $t$ for Test Problem 4.3.

$$
\begin{aligned}
& \beta_{0}=\frac{\left|\mathscr{Z}_{1}(\xi, t, p)\right|}{\left|\mathscr{E}_{0}(\xi, t, p)\right|}=0.027000162277578<1, \\
& \beta_{1}=\frac{\left|\mathscr{E}_{2}(\xi, t, p)\right|}{\left|\mathscr{Z}_{1}(\xi, t, p)\right|}=3.746191596095180 e-04<1, \\
& \beta_{2}=\frac{\left|\mathscr{Z}_{3}(\xi, t, p)\right|}{\left|\mathscr{E}_{2}(\xi, t, p)\right|}=3.370330110176276 e-06<1, \\
& \beta_{3}=\frac{\left|\mathscr{Z}_{4}(\xi, t, p)\right|}{\left|\mathscr{Z}_{3}(\xi, t, p)\right|}=2.274994159334152 e-08<1,
\end{aligned}
$$

which shows that for $n \geq 0$, the values of $\beta_{n}$ are less than one, which provide the proof as well that the proposed algorithm (MVIA-II) is convergent.

The numerical results of the MVIA-II for Test Problem 4.3 are reported in Table 4 along with the exact solution and the results in the recent literature. It can be noticed from the table that the MVIA-II gives more accurate results in comparison with the approaches given in $[15,48]$.

The accuracy of the suggested MVIA-II is also verified from Figure 4, whereas in Figure 5, we have shown the absolute error for various time instants $t=0.3, t=0.5$, and $t=1.0$.

Figure 5 is the evidence of better accuracy of the proposed MVIA-II.

\section{Conclusion}

The primal purpose of this paper is to study convergence analysis of a modified variational iteration algorithm-II (MVIA-II) and its applications in physical and biological sciences. The numerical results and theoretical study of the convergence analysis point out that the proposed algorithm can solve nonlinear problems efficiently and accurately. The numerical, as well as graphical results, generated by the MVIA-II are compared with the results of the compact finite difference method, the second kind Chebyshev wavelets procedure, and a procedure based on Hermite interpolant multiscaling functions, which revealed that the MVIA-II is of high accuracy and yields accurate results. This modified algorithm facilitates computational work for solving linear as well as nonlinear problems arising in engineering and applied sciences. High-accuracy solutions can be achieved in a few iterations of the proposed algorithm compared to earlier methods reported in the literature. We hope that the achieved results will be useful for further studies in scientific research, especially in physical and biological sciences.

\section{Data Availability}

Data will be provided on request to the first author.

\section{Conflicts of Interest}

The authors declare that they have no conflicts of interest. 


\section{Acknowledgments}

The research was supported by the National Natural Science Foundation of China (Grant nos. 11971142, 11871202, $61673169,11701176,11626101$, and 11601485).

\section{References}

[1] X.-X. Li and J.-H. He, "Along the evolution process kleiber's 3/4 law makes way for rubner's surface law: a fractal approach," Fractals, vol. 27, no. 02, Article ID 1950015, 2019.

[2] K. M. Owolabi, "High-dimensional spatial patterns in fractional reaction-diffusion system arising in biology," Chaos, Solitons \& Fractals, vol. 134, Article ID 109723, 2020.

[3] J. Ul Rahman, D. Lu, M. Suleman, J.-H. He, and M. Ramzan, "He-elzaki method for spatial diffusion of biological population," Fractals, vol. 27, no. 05, Article ID 1950069, 2019.

[4] E. A. Saied, "The non-classical solution of the inhomogeneous non-linear diffusion equation," Applied Mathematics and Computation, vol. 98, no. 2-3, pp. 103-108, 1999.

[5] M. El-Gamel, "A wavelet-galerkin method for a singularly perturbed convection-dominated diffusion equation," $A p$ plied Mathematics and Computation, vol. 181, no. 2, pp. 1635-1644, 2006.

[6] Ş. Yüzbaşı and N. Şahin, "Numerical solutions of singularly perturbed one-dimensional parabolic convection-diffusion problems by the Bessel collocation method," Applied Mathematics and Computation, vol. 220, no. 1, pp. 305-315, 2013.

[7] W. Wen-qia, "The alternating segment Crank-Nicolson method for solving convection-diffusion equation with variable coefficient," Applied Mathematics and Mechanics, vol. 24, no. 1, pp. 32-42, 2003.

[8] S. Dhawan, S. Kapoor, and S. Kumar, "Numerical method for advection diffusion equation using FEM and B-splines," Journal of Computational Science, vol. 3, no. 5, pp. 429-437, 2012.

[9] J. I. Ramos, "A piecewise-analytical method for singularly perturbed parabolic problems," Applied Mathematics and Computation, vol. 161, no. 2, pp. 501-512, 2005.

[10] S. Karaa and J. Zhang, "High order ADI method for solving unsteady convection-diffusion problems," Journal of Computational Physics, vol. 198, no. 1, pp. 1-9, 2004.

[11] C. Bota and B. Căruntu, "Approximate analytical solutions of nonlinear differential equations using the least squares homotopy perturbation method," Journal of Mathematical Analysis and Applications, vol. 448, no. 1, pp. 401-408, 2017.

[12] M. M. Gupta, J. Kouatchou, and J. Zhang, "A compact multigrid solver for convection-diffusion equations," Journal of Computational Physics, vol. 132, no. 1, pp. 123-129, 1997.

[13] L. Li, R. Mei, and J. F. Klausner, "Lattice Boltzmann models for the convection-diffusion equation: D2q5 vs d2q9," International Journal of Heat and Mass Transfer, vol. 108, pp. 41-62, 2017.

[14] R. Becker and B. Vexler, "Optimal control of the convectiondiffusion equation using stabilized finite element methods," Numerische Mathematik, vol. 106, no. 3, pp. 349-367, 2007.

[15] F. Zhou and X. Xu, "Numerical solution of the convection diffusion equations by the second kind Chebyshev wavelets," Applied Mathematics and Computation, vol. 247, pp. 353-367, 2014.

[16] D. Shi, J. Chen, and X. Wang, "Low order nonconforming expanded characteristic- mixed finite element method for the convection- diffusion problem," Applied Mathematics \& Information Sciences, vol. 7, no. 1, pp. 67-74, 2013.
[17] J. B. Munyakazi, "A robust finite difference method for twoparameter parabolic convection-diffusion problems," Applied Mathematics \& Information Sciences, vol. 9, no. 6, p. 2877, 2015.

[18] A.-M. Wazwaz, "Exact solutions to nonlinear diffusion equations obtained by the decomposition method," Applied Mathematics and Computation, vol. 123, no. 1, pp. 109-122, 2001.

[19] G. Gürarslan, "Numerical modelling of linear and nonlinear diffusion equations by compact finite difference method," Applied Mathematics and Computation, vol. 216, no. 8, pp. 2472-2478, 2010.

[20] J. Li, J. Zhao, L. Qian, and X. Feng, "Two-level meshless local Petrov Galerkin method for multi-dimensional nonlinear convection-diffusion equation based on radial basis function," Numerical Heat Transfer, Part B: Fundamentals, vol. 74, no. 4, pp. 685-698, 2018.

[21] C. Cancès, C. Chainais-Hillairet, and S. Krell, "Numerical analysis of a nonlinear free-energy diminishing discrete duality finite volume scheme for convection diffusion equations," Computational Methods in Applied Mathematics, vol. 18, no. 3, pp. 407-432, 2018.

[22] S. Srinivasan, J. Poggie, and X. Zhang, "A positivity-preserving high order discontinuous Galerkin scheme for convection-diffusion equations," Journal of Computational Physics, vol. 366, pp. 120-143, 2018.

[23] R. Costa, J. M. Nóbrega, S. Clain, G. J. Machado, and R. Loubère, "Very high-order accurate finite volume scheme for the convection-diffusion equation with general boundary conditions on arbitrary curved boundaries," International Journal for Numerical Methods in Engineering, vol. 117, no. 2, pp. 188-220, 2019.

[24] J.-H. He, "Variational iteration method - a kind of non-linear analytical technique: some examples," International Journal of Non-linear Mechanics, vol. 34, no. 4, pp. 699-708, 1999.

[25] J.-H. He, G.-C. Wu, and F. Austin, "The variational iteration method which should be followed," Nonlinear Science Letters A, vol. 1, no. 1, pp. 1-30, 2010.

[26] H. Ahmad and T. A. Khan, "Variational iteration algorithm i with an auxiliary parameter for the solution of differential equations of motion for simple and damped mass-spring systems," Noise \& Vibration Worldwide, vol. 51, no. 1-2, pp. 12-20, 2020.

[27] H. Ahmad, T. A. Khan, P. S. Stanimirovic, and I. Ahmad, "Modified variational iteration technique for the numerical solution of fifth order kdv type equations," Journal of Applied and Computational Mechanics, vol. 6, 2020.

[28] C. Chun, "Variational iteration method for a reliable treatment of heat equations with ill-defined initial data," International Journal of Nonlinear Sciences and Numerical Simulation, vol. 9, no. 4, pp. 435-440, 2008.

[29] J. Saberi-Nadjafi and M. Tamamgar, "The variational iteration method: a highly promising method for solving the system of integro-differential equations," Computers \& Mathematics with Applications, vol. 56, no. 2, pp. 346-351, 2008.

[30] R. Mokhtari, "Variational iteration method for solving nonlinear differential-difference equations," International Journal of Nonlinear Sciences and Numerical Simulation, vol. 9, no. 1, pp. 19-24, 2008.

[31] İ. Ateş and A. Yildirim, "Application of variational iteration method to fractional initial-value problems," International Journal of Nonlinear Sciences and Numerical Simulation, vol. 10, no. 7, pp. 877-884, 2009. 
[32] N. Herisanu and V. Marinca, "A modified variational iteration method for strongly nonlinear problems," Nonlinear Science Letters A, vol. 1, no. 2, pp. 183-192, 2010.

[33] E. Hesameddini and H. Latifizadeh, "Reconstruction of variational iteration algorithms using the laplace transform," International Journal of Nonlinear Sciences and Numerical Simulation, vol. 10, no. 11-12, pp. 1377-1382, 2009.

[34] D. K. Salkuyeh, "Convergence of the variational iteration method for solving linear systems of odes with constant coefficients," Computers \& Mathematics with Applications, vol. 56, no. 8, pp. 2027-2033, 2008.

[35] E. Yilmaz and M. Inc, "Numerical simulation of the squeezing flow between two infinite plates by means of the modified variational iteration method with an auxiliary parameter," Nonlinear Science Letters A, vol. 1, no. 3, pp. 297-306, 2010.

[36] H. Ahmad and T. A. Khan, "Variational iteration algorithm-i with an auxiliary parameter for wave-like vibration equations," Journal of Low Frequency Noise, Vibration and Active Control, vol. 38, no. 3-4, pp. 1113-1124, 2019.

[37] H. Ahmad, A. R. Seadawy, T. A. Khan, and P. Thounthong, "Analytic approximate solutions for some nonlinear parabolic dynamical wave equations," Journal of Taibah University for Science, vol. 14, no. 1, pp. 346-358, 2020.

[38] H. Ahmad, A. R. Seadawy, and T. A. Khan, "Study on numerical solution of dispersive water wave phenomena by using a reliable modification of variational iteration algorithm," Mathematics and Computers in Simulation, vol. 177, pp. 13-23, 2020.

[39] J.-H. He, "Notes on the optimal variational iteration method," Applied Mathematics Letters, vol. 25, no. 10, pp. 1579-1581, 2012.

[40] H. Ghaneai and M. M. Hosseini, "Variational iteration method with an auxiliary parameter for solving wave-like and heat-like equations in large domains," Computers \& Mathematics with Applications, vol. 69, no. 5, pp. 363-373, 2015.

[41] H. Ahmad, "Variational iteration method with an auxiliary parameter for solving differential equations of the fifth order," Nonlinear Science Letters A, vol. 9, no. 1, pp. 27-35, 2018.

[42] D.-N. Yu, J.-H. He, and A. G. Garcia, "Homotopy perturbation method with an auxiliary parameter for nonlinear oscillators," Journal of Low Frequency Noise, Vibration and Active Control, vol. 38, no. 3-4, 2019.

[43] H. Ahmad, T. A. Khan, H. Durur, G. Ismail, and A. Yokus, "Analytic approximate solutions of diffusion equations arising in oil pollution," Journal of Ocean Engineering and Science, In press, 2020.

[44] M. Inokuti, H. Sekine, and T. Mura, "General use of the Lagrange multiplier in nonlinear mathematical physics," Variational Method in the Mechanics of Solids, vol. 33, no. 5, pp. 156-162, 1980.

[45] Z. M. Odibat, "A study on the convergence of variational iteration method," Mathematical and Computer Modelling, vol. 51, no. 9-10, pp. 1181-1192, 2010.

[46] H. Ghaneai and M. Hosseini, "Solving differential-algebraic equations through variational iteration method with an auxiliary parameter," Applied Mathematical Modelling, vol. 40, no. 5-6, pp. 3991-4001, 2016.

[47] J.-H. He and C. Sun, "A variational principle for a thin film equation," Journal of Mathematical Chemistry, vol. 57, no. 9, pp. 2075-2081, 2019.

[48] E. Ashpazzadeh and M. Lakestani, "Hermite interpolant multiscaling functions for numerical solution of the convection diffusion equations," Boletim da Sociedade Paranaense de Matemática, vol. 36, no. 2, pp. 83-97, 2018. 